\title{
Financial distress and its associated burden in couples coping with an advanced cancer
}

\section{Juliet Kroll ( $\nabla$ jlkroll@mdanderson.org )}

The University of Texas MD Anderson Cancer Center https://orcid.org/0000-0002-8873-7239

\section{Seokhun Kim}

The University of Texas MD Anderson Cancer Center

\section{Dalnim Cho}

The University of Texas MD Anderson Cancer Center

\section{Shiao-Pei Weathers}

The University of Texas MD Anderson Cancer Center

\section{Aileen Chen}

The University of Texas MD Anderson Cancer Center

\section{Grace Smith}

The University of Texas MD Anderson Cancer Center

\section{Eduardo Bruera}

The University of Texas MD Anderson Cancer Center

\section{Kathrin Milbury}

The University of Texas MD Anderson Cancer Center

\section{Research Article}

Keywords: financial distress, couples, advanced cancer, end-of-life, physical quality of life

Posted Date: June 21st, 2021

DOl: https://doi.org/10.21203/rs.3.rs-566445/v1

License: (c) (1) This work is licensed under a Creative Commons Attribution 4.0 International License.

Read Full License

Version of Record: A version of this preprint was published at Supportive Care in Cancer on February 3rd, 2022. See the published version at https://doi.org/10.1007/s00520-021-06758-w. 


\section{Abstract \\ Purpose}

As the economic burden and financial distress (FD) resulting from cancer care are increasingly recognized, FD remains inadequately understood from the perspective of patients and their spousal caregiver, the relational context where most financial and treatment decisions are navigated. Therefore, we assessed FD in both patients with advanced cancer and their spouses to identify symptom and QOL correlates. We additionally examined if illness communication moderated the association between FD and QOL.

\section{Methods}

Patients undergoing treatment for stage III/IV lung cancer or grade III/IV primary brain tumor and their spouses completed measures of their own FD, QOL, symptoms, perception of their spouse's symptoms, and overall illness communication.

\section{Results}

Patients (62.7\%) and spouses (64.7\%) endorsed FD; however, spouses rated FD with greater relative severity. For both, FD was associated with greater anxiety, depression, and poorer physical QOL. For patients, FD was additionally associated with poorer mental QOL. Spousal caregivers accurately perceived patient FD, yet patients underreported spouse's FD by a clinically meaningful difference. A 3way interaction (FD X role X illness communication) revealed $(b=.40, p=.041)$ that illness communication moderated the association between FD and physical QOL for spouses only.

\section{Conclusion}

In the advanced cancer setting, FD is prevalent in both patients and their caregivers and associated with psychological distress and poor physical QOL. Results suggest that optimal FD interventions should include patients and spouses. As illness communication appears to buffer the negative association of FD with physical QOL, studies targeting illness communication deficits in couples facing advanced disease are warranted.

\section{Introduction}

The burden of a cancer is not only experienced by the patient, but also by family members increasingly involved in caregiving roles throughout treatment and at end-of-life. Among family caregivers, the spouse or romantic partner (hereafter referred to as spouse or spousal caregiver) often serves as the primary source of both instrumental and emotional support to the patient [1]. This provision of support does not 
come without challenges - widely conceptualized as "caregiving burden"[2]. Caregiving burden is particularly high in those caring for a patient with an advanced/incurable cancer [3] and is associated with poorer physical and psychological health of the caregiver. As one might imagine, caregiving burden is additionally associated with poorer patient quality of life (QOL) [1]. A spouses' psychological state often impacts that of their patient, and vice versa, resulting in an interdependence of both psychological state and QOL among couples facing cancer [4; 5]. Physical health is additionally observed to be interdependent in couples [6], yet not all studies find this association in cancer patients [7]. This interdependence reaffirms the value of capturing both the patient and spousal caregiver's perspectives, in that the physical or mental health status of the spousal caregiver may indicate greater risk of psychological distress or poorer physical QOL for their partner and vice versa.

One increasingly identified consequence of cancer treatment that impacts both the patient and their spousal caregiver is financial distress (FD), the subjective psychological experience of financial concerns. FD is one unique component of financial toxicity (FT) which, additionally captures the objective material financial burden (e.g. co-pays and out-of-pocket costs) and behavioral consequences (e.g. lost job) with cancer treatment [8]. FT is estimated to rise with ever increasing cancer costs and out of pocket patient expenses [9], likely resulting in continued deleterious impact on cancer care and patient outcomes [10; 11]. In the palliative care setting, FD may be particularly relevant. Length of treatment and worse overall health and functional status are associated with greater objective financial burden [12], and temporal patterns of cancer costs typically follow a "u-shaped" curve with highest burden at diagnosis and end-oflife [13]. Moreover, financial toxicity is recently reported at severe levels in up to $18 \%$ of patients over 70 years of age with advanced cancer, and further associated with greater general psychological distress and lower health related QOL [14]. Longitudinal studies in patients with metastatic disease additionally find that major financial hardship is experienced by $24.6 \%$ of patients 3 months after diagnosis and increases to a staggering $71.5 \% 12$ months following a diagnosis [15]. Accumulating research illuminates the deleterious impact of objective economic burden experienced by cancer patients [16;12], spousal caregivers independently [17; 18; 19], and the pediatric patient-parent caregiver dyad [20]. However, FD from the patient-spousal caregiver dyadic perspective remains surprisingly unexplored in cancer patients.

As treatment goals for patients with incurable disease often aim to prolong life while maximizing QOL, financial distress is a highly relevant topic to QOL that has until recently, received little attention in supportive care settings. At an individual level in patients with advanced cancer, FD is associated with greater somatic symptom burden (e.g. ratings on the Edmonton Symptom Assessment Scale (ESAS), symptoms of anxiety, depression, etc.) in both national and international samples [21;22]. As members of a romantic couple often have unique psychological responses to a shared stressor [23], it is plausible that even though the objective economic burden is shared, the subjective psychological experience of FD may be distinctly experienced by each member of the couple. Therefore, the goal of the present study is to assess the intensity and relative severity of FD in patients and their spousal caregivers and further identify associated symptom correlates (particularly QOL) as a first step to understand how couples approach this concern. In effort to identify targets for future interventions, we will explore if illness communication moderates the relation between FD and mental and physical QOL using dyadic analyses. 


\section{Method}

\section{Participants}

In the present study, 76 adult patients with a diagnosis of Stage III-b or IV non-small cell lung cancer (NSCLC) or Grade III or IV primary brain tumor undergoing treatment (e.g. chemotherapy, surgery, and/or radiotherapy) and their spousal caregiver (e.g. spouse or romantic partner with whom patient has cohabitated $>6$ months) were enrolled from a large comprehensive cancer center. Patients with cognitive deficits, as indicated by their clinical team, were excluded. All participants were able to read, write and speak English.

\section{Procedures}

Participants were identified through the electronic health record (EHR) and recruited in their respective clinics by research staff. Participants were further screened at their clinic visit and, if eligible, provided written informed consent. If the spousal caregiver was not present at the initial visit, permission was obtained from the patient for the research team to contact the caregiver by phone to obtain consent. We approached 100 consecutive eligible dyads and 76 consented. Primary reasons for patients to decline participation was "lack of interest" and being "too busy." Participants completed a series of validated questionnaires by pen and paper. The University of Texas MD Anderson Cancer Center Institutional Review Board approved the study, and all participants were compensated for their time.

\section{Measures}

Demographics and medical details. Participants provided demographic information including age, sex, race/ethnicity, employment status, education and type of relationship with spousal caregiver (e.g. married or domestic partner). Disease stage/grade and time since diagnosis were extracted from the EHR.

Financial Distress (FD). FD was captured by the Edmonton Symptom Assessment Scale (ESAS), a 12item measure of common symptoms where patients rate their symptoms on a 0-10 Likert-type scale, with 10 indicating greatest severity of symptom [24]. Topics range from appetite, to pain, to spiritual pain. FD was extracted as a single item from the ESAS total. Participants rated both the severity of their own FD and their perception of their spouse's symptom severity.

Psychological distress. Participants completed self-report questionnaires individually to assess their psychological well-being. This included the Brief Symptom Inventory (BSI-18), an 18-item measure which includes a subscale capturing symptoms of anxiety [25], which is reported here. Participants rate their experience of symptoms over the past week, on a 0-4 scale of severity of distress ranging from not at all to extremely. Depression over the past week was additionally captured by the Center for Epidemiological Studies-Depression (CES-D), a 20-item measure where participants rate the frequency of experiencing depressive symptoms on a 1-4 scale ranging from almost never to almost always (5 to 7 days). Scores greater than 16 indicate likely case of clinical depressive episode [26]. 
Mental and Physical QOL. Mental and physical QOL were assessed with the 10-item PROMIS Global Health short form, where participants rate QOL on a 5-point Likert type scale ranging from 1 (poor) to 5 (excellent). The final item subscales were scored for both global physical QOL and global mental QOL [27].

Illness Communication. Each participant's perceived ease of illness communication within the couple was captured with the Couples' Illness Communication Scale (CICS), a brief 4-item self-report measure [28]. Scores range from 1-20 with lower scores indicating perceptions of poorer illness communication within the couple.

\section{Analytic Plan}

First, descriptive statistics, paired sample t-tests for continuous, and chi-squared tests for categorical variables were calculated to examine group differences between patients and spouses across all variables. Any skewed items were transformed prior to analyses. Second, bivariate Pearson correlations among variables of interest were run separately for patients and spousal caregivers. Third, to examine if illness communication moderated the associations between FD and mental QOL and physical QOL, we conducted separate dyadic analyses using a multi-level modeling (MLM) approach to account for our nested data structure (individuals within dyads) [29]. As disease site, age, sex, education and employment status have all been previously associated with objective financial burden [12], dyadic analyses controlled for these covariates. For ease of interpretation, illness communication (CICS) was grand mean centered. Full Information Maximum Likelihood method was used to deal with missing data in the outcomes. SPSS 24 was used for descriptive statistics and correlations. Stata 16 was used for dyadic analyses.

\section{Results}

\section{Descriptive statistics}

A total of 156 participants (e.g. 76 couples) participated in the study. For patients, mean age was 58.19 years $(S D=12.31)$, approximately half were female $(50.7 \%)$ and the majority had opposite sex spouse ( $n=3$ had same-sex spouse). Most patients were well-educated (44.4\% college degree), nonHispanic White (81.4\%) and retired (46.5\%) (See Table 1). For spouses, mean age was 57.83 years $(S D=13.12)$. Spouses were also well-educated ( $49.6 \%$ college graduate), non-Hispanic White $(80.3 \%)$, and half of the sample was full-time employed (50.7\%). Patients reported mild-to-moderate levels of present psychological distress (BSI-18: $M=0.31, S D=0.34$; CESD: $M=10.69, S D=7.3$ ), with spouses reporting greater anxiety (BSI-18: $M=0.46, S D=0.45 ; t=2.77, p=0.007$ ). Both patients and their spouses reported high illness communication (CICS: $M=16.74, S D=3.1 ; M=15.67, S D=3.6$ respectively), which was greater in patients $(t=2.38, p=.040)$. As expected, patients reported poorer physical QOL than their spouses $(t=-3.01, p=.003)$, yet there was no group difference in mental QOL. See Table 1.

\section{Frequency and Relative Severity of FD}


In patients, $62.7 \%$ reported presence of FD (defined as scores of 1 or greater) and $24 \%$ reported high FD (defined as score of 4 or greater according to Barbaret et al., (30)). In spousal caregivers, $64.7 \%$ reported presence of FD and $32.3 \%$ reported high FD. On average, patients and spousal caregivers reported mild levels of $\mathrm{FD}(M=2.32, S D=2.81, M=2.81, S D=3.12$ respectively), where scores $1-3$ specify mild distress. Among the 12 common cancer symptoms assessed, patients rated FD as the 7th most severe symptom. In turn, spouses rated their own FD as the 2nd most severe symptom (after sleep disturbances). See Table 2 and Figure 1 for severity ratings across ESAS.

\section{Symptoms associated with FD}

For patients, FD was associated with greater depressive symptoms ( $r=.29, p=.010)$, anxiety $(r=.29$, $p=.010)$, and poorer physical QOL $(r=-.25, p=.030)$. For spouses, FD was associated with greater depressive symptoms $(r=0.39, p=.001)$, anxiety $(r=0.31, p=.010)$, poorer physical QOL $(r=-0.38, p=.002)$ and poorer mental QOL $(r=-0.44, p<.001)$. Table 3 presents correlations among major study variables by role (i.e. patient, spousal caregiver).

\section{Self-report and Partner Proxy-report of FD}

While spousal caregivers accurately rated the FD of their patient, patients tended to underperceive the extent of their spouse's experience of FD by an entire point $(t=2.80, p=.007)$, where a 1-point difference indicates a clinically significant change. As expected, patients' and spouses' self-report of FD were correlated $(r=.53, p=<.001)$, controlling for couple membership. See Tables 4 and 5 for comparison between self-report and spouse proxy-report across all ESAS symptoms.

\section{Symptoms associated with Illness Communication}

For both patients and spouses, greater comfort with illness communication was associated with fewer symptoms of anxiety, ( $r=-.239, p=.046 ; r=-.371, p=.001$, respectively). For spouses only, greater comfort with illness communication was additionally associated with fewer symptoms of depression ( $r=-.382$, $p=.001)$ along with greater physical $(r=.271, p<.001)$ and mental QOL $(r=.326, p=.005)$. FD was not correlated with illness communication for either member of the couple (see Table 3). Overall, patients' and spouses' report of comfort with illness communication were moderately correlated, $r=.361, p=.002$, controlling for couple membership.

\section{Dyadic Models of FD}

Physical QOL. MLM analyses revealed that the association of FD with physical QOL significantly varied by role and across illness communication (FD X role X illness communication) ( $b=.37, p=.039$ ), (Figure 2). When illness communication was low (-1 SD from its mean), spouses demonstrated a stronger association between FD and physical QOL $(b=-2.08, p<.001)$ than when their illness communication was high ( +1 SD from its mean) $(b=.49, p=.508)$. 
For patients, the negative association between FD and physical QOL did not differ if patients were high $(b=-1.15, p=.05)$ or low in illness communication $(b=-1.18, p=.06)$. (See Figure 2 for graphical representation)

Mental QOL. No three-way interaction, two-way interactions, or main effects were found among FD, mental QOL, and illness communication in either patients or spousal caregivers for this sample.

\section{Discussion}

This cross-sectional survey was designed to assess the prevalence, intensity and relative severity of FD along with any associated symptoms of psychological distress and QOL in both patients with advanced cancer and their spousal caregivers. Whereas the objective material financial burden and the subjective psychological experience of FD has been studied in a variety of advanced cancer settings; to the best of our knowledge, this is the first study to examine FD at the analysis level of the couple - the relationship where financial concerns and caregiving are most intimately navigated.

\section{Prevalence and severity of FD}

FD was present in over $60 \%$ of the sample and at high levels in $24 \%$ of patients and $34 \%$ of caregivers, reinforcing the relevance of the subjective psychological experience of financial distress - even for families with insurance and treated at comprehensive cancer centers who may experience less of the objective material financial burden compare to those without insurance. Indeed, over half of the spousal caregivers continued to work full time and $35 \%$ had reached retirement. On average, patients and spousal caregivers reported mild FD and were concordant in their intensity ratings. Compared to patients with advanced cancer, where estimates of FD include patients with and without spousal caregivers [30; 18], the prevalence of FD and high FD were lower in this partnered sample. As previous studies identify unpartnered status is a significant predictor of FD in advanced cancer patients [30], lower rates of FD in the present study may be attributable to the partnered nature of this sample where a spouse may be available for financial and/or emotional support.

Regarding relative severity, FD was rated as the second highest concern for spousal caregivers and the seventh highest symptom (out of twelve total) for patients. As patients are expected to experience physical side effects from treatment and spousal caregivers will eventually survive the patient with incurable disease amounting to a longer period of financial obligations, the difference in relative severity of FD is not surprising. What is surprising, is that despite the general tendency of spousal caregivers to overestimate symptoms in their patients [31] (and who did so here on 7 out of 12 symptoms) spousal caregivers were accurate in estimating the extent of the patient's FD (along with appetite, well-being, and shortness of breath). On the other hand, patients tended to underestimate their spousal caregiver's FD by up to 1 point on the ESAS, which indicates a clinically meaningful difference. It is possible that patients are more cognitively focused on their own present symptom burden and find it difficult to imagine the future orientated financial concerns of their spouse. Additionally, spousal caregivers may limit raising any apprehension regarding present or future financial responsibilities directly with the patient out of concern 
for adding additional stressors. In fact, this intended "protective buffering" is well documented in couples coping with cancer across a variety of topics both patient to caregivers and caregiver to patient [32; 33]. While perceived illness communication within the couple was reported at high rates by both patients and spouses, the extent to which couples discussed finances was not directly assessed.

\section{Symptoms associated with FD}

Despite general ratings of mild FD, FD in patients was associated with greater symptoms of anxiety and depression as well as poorer physical QOL, consistent with other studies of FD $[34 ; 21 ; 22]$ and objective financial burden $[11 ; 17 ; 14 ; 19 ; 18]$. This highlights that even at mild intensity, FD is associated with other domains of patient distress. What we observed for the first time, is that the FD of spousal caregivers is additionally associated with greater symptoms of anxiety, depression and poorer physical QOL. While cross sectional in nature, the associated symptom and QOL correlates suggest that the subjective financial distress not only an important component of patient experience but a unique component of caregiver burden. Longitudinal analyses will be necessary to identify if financial distress predicts any exacerbation of pre-existing symptoms of anxiety, depression or poor QOL in spouses. As interventions designed to target financial toxicity (i.e. material objective burden, psychological distress, and behavioral consequences) of cancer care are only in their infancy, the findings here underscore the importance of including the spousal caregiver both in the assessment and as a target of intervention.

\section{Moderating Effects of Illness Communication}

Dyadic analyses revealed a moderating effect of illness communication on the association between FD and physical QOL, suggesting that greater comfort with illness communication may have a protective effect against the negative association of FD with physical QOL in spouses. While lower levels of illness communication did not appear to be directly associated with greater FD, spouses with lower levels of illness communication did appear to have a stronger relation between FD and physical QOL. Prospective study is needed to identify any directional associations. However, seminal studies in couples coping with cancer identify that the avoidance of cancer-related communication is associated with negative consequences of both patient and spousal wellbeing, and in turn mutual constructive communication is associated with less psychological distress [33]. Here, we observe the potential of a similarly beneficial pattern of illness communication in couples facing an incurable cancer - that it may "buffer" against the consequences of FD. Interestingly, this was only observed in spouses. As spouses appear to experience greater relative burden of FD than patients, there may be greater variability to detect associations in this sample.

\section{Limitations and future directions}

FD was measured consistently with previously published studies [21;30; 31], providing an opportunity to compare among samples. However, there is no standardized measurement tool for FD (in contrast to measures for financial toxicity) and a validated measure would be preferable. As the study of FD continues to develop, generation of a brief psychometrically valid tool for the subjective psychologically 
experience of financial distress is warranted. The study was additionally limited in the cross-sectional design, which precludes any directional or causal inferences. Further, the comprehensive cancer center sample naturally limits the generalizability of these findings and highlights the urgent need for future studies in settings of uninsured patients and their spousal caregivers. As the material financial burden is undoubtedly greater in uninsured patients, continued study on the distinct experience the subjective financial distress of cancer care is warranted. Despite the present limitations, these first findings at the level of the couple help characterize impact of FD on both the patient and the spouse and identify potential targets for intervention. Ongoing and published studies designed to mitigate the financial burden of cancer most commonly include financial education, access to financial navigation, or even tangible caregiving skills $[35 ; 36]$. Findings presented here underscore that optimal FD interventions in the advanced cancer setting should include both patient and spouses and identify illness communication as a potential component for intervention study. As illness communication appears to buffer the negative association of FD with QOL for spouses, future study targeting deficits illness communication appear to be warranted. In the context of illness communication, it will also be valuable to directly assess communication as related to financial concerns and control for objective income to further the conceptualization of subjective psychological financial distress compared to the objective material financial burden.

\section{Clinical implications}

The present study underscores the importance of assessing financial distress in both patients with advanced cancer and their spousal caregiver. Even at a mild symptom presentation, FD is associated with greater psychological distress and poorer physical QOL in both the patient and their spouse. Further, FD appears to be of greater relative severity to spouses than patients, and patients tended to underreport their spouses FD by a clinically meaningful difference. As patients approach end-of-life, the spousal caregiver is often the de-facto treatment decision maker and therefore an appropriate party to support. For spouses with deficits in illness communication, increased comfort with illness communication may help to buffer the burden of FD on physical QOL. However, it remains unknown if manipulation of illness communication (i.e. strategies to increase communication) will lead to improvement in spouse's physical QOL. As interventions are designed to address FD, findings here underscore that optimal interventions should include the perspective of both the patient and their spousal caregiver.

\section{Declarations}

Funding. A portion of Dr. Kroll's work on this manuscript was supported by funding from the Cancer Prevention and Research Institute of Texas (RP170259). A portion of Dr. Cho's work on this manuscript was supported by funding from the Department of Defense (W81XWH-19-1-0460).

Conflicts of interest/Competing interests. The authors JLK, SK, DC, SW, ABC, GS, EB, KM have no conflicts of interest to disclose 
Availability of data and material. Data will be made available upon reasonable request by contacting primary author.

Code availability. Not applicable

Authors' contributions: JLK, KM, EB, and GS conceptualized the study. JK, SK, DC analyzed the data. SW, $A B C$ were involved with data collection. All authors contributed to writing of the manuscript.

Ethics approval. This study was approved by the MD Anderson Cancer Center Institutional Review Board.

Consent to participate. All participants provided written informed consent prior to participation in this study.

Consent for publication. All authors contributed to this work and reviewed final version.

\section{References}

1. Kim Y, Kashy DA, Wellisch DK, Spillers RL, Kaw CK, Smith TG. Quality of life of couples dealing with cancer: dyadic and individual adjustment among breast and prostate cancer survivors and their spousal caregivers. Ann Behav Med. 2008;35(2):230-238. doi:10.1007/s12160-008-9026-y.

2. Fletcher BS, Miaskowski C, Given B, Schumacher K. The cancer family caregiving experience: an updated and expanded conceptual model. Eur J Oncol Nurs.

2012;16(4):387-398. doi:10.1016/j.ejon.2011.09.001.

3. Clark MM, Rummans TA, Sloan JA, et al. Quality of life of caregivers of patients with advanced-stage cancer. Am J Hosp Palliat Care. 2006;23(3):185-191.

doi:10.1177/1049909106289074.

4. Jacobs JM, Shaffer KM, Nipp RD, et al. Distress is Interdependent in Patients and Caregivers with Newly Diagnosed Incurable Cancers. Ann Behav Med. 2017;51(4):519-531. doi:10.1007/s12160-0179875-3.

5. Milbury K, Badr H, Fossella F, Pisters KM, Carmack CL. Longitudinal associations between caregiver burden and patient and spouse distress in couples coping with lung

cancer. Support Care Cancer. 2013;21(9):2371-2379. doi:10.1007/s00520-013-1795-6.

6. Meyler D, Stimpson JP, Peek MK. Health concordance within couples: a systematic review. Soc Sci Med. 2007;64(11):2297-2310. doi:10.1016/j.socscimed.2007.02.007 
7. Streck BP, Wardell DW, LoBiondo-Wood G, Beauchamp JES. Interdependence of physical and psychological morbidity among patients with cancer and family caregivers: Review of the literature. Psychooncology. 2020;29(6):974-989. doi:10.1002/pon.5382

8. National Cancer Institute. Financial Hardship [cited 2020 March 3]. Available from:

https://healthcaredelivery.cancer.gov/hardship/.

9. Mariotto AB, Enewold L, Zhao J, Zeruto CA, Yabroff KR. Medical Care Costs Associated with Cancer Survivorship in the United States. Cancer Epidemiol Biomarkers Prev June 102020 DOI: 10.1158/10559965.EPI-19-1534.

10. Zafar SY. Financial Toxicity of Cancer Care: It's Time to Intervene. J Natl Cancer Inst. 2015;108(5):djv370. Published 2015 Dec 11. doi:10.1093/jnci/djv370.

11. Zullig LL, Peppercorn JM, Schrag D, Taylor DH, Jr., Lu Y, Samsa G, Abernethy AP, Zafar SY. Financial Distress, Use of Cost-Coping Strategies, and Adherence to Prescription Medication Among Patients With Cancer. Journal of oncology practice. 2013;9(6S):60s-3s. doi: 10.1200/JOP.2013.000971. PubMed PMID: 29431038; PMCID: 3825170.

12. Smith GL, Lopez-Olivo MA, Advani PG, et al. Financial Burdens of Cancer Treatment: A Systematic Review of Risk Factors and Outcomes. Journal of the National Comprehensive Cancer Network: JNCCN 2019;17:1184-9

13. Yabroff KR, Lund J, Kepka D, Mariotto A. Economic burden of cancer in the United States: estimates, projections, and future research. Cancer Epidemiol Biomarkers Prev. 2011;20(10):2006-2014. doi:10.1158/1055-9965.EPI-11-0650.

14. Arastu A, Patel A, Mohile SG, et al. Assessment of Financial Toxicity Among Older Adults with Advanced Cancer. JAMA Netw Open. 2020;3(12):e2025810. doi:10.1001/jamanetworkopen.2020.25810.

15. Shankaran V, Unger JM, Darke A, Suga JM, Wade JL, Kourlas P, Sreenivasa R Chandana SR, O'Rourke MA, Satti S, Liggett D, Hershman DL, and Ramsey SD. Cumulative incidence of financial hardship in metastatic colorectal cancer patients: Primary endpoint results for SWOG S1417CD. Journal of Clinical Oncology 2020 38:15_suppl, 7010-7010.

16. Ramsey SD, Bansal A, Fedorenko CR, et al. Financial Insolvency as a Risk Factor for Early Mortality Among Patients With Cancer. Journal of clinical oncology: official journal of the American Society of Clinical Oncology 2016;34:980-6.

17. Van Houtven $\mathrm{CH}$, Ramsey SD, Hornbrook MC, Atienza AA, van Ryn M. Economic burden for informal caregivers of lung and colorectal cancer patients. Oncologist. 2010;15(8):883-893.

doi:10.1634/theoncologist.2010-0005. 
18. Li C, Zeliadt SB, Hall IJ, et al. Burden among partner caregivers of patients diagnosed with localized prostate cancer within 1 year after diagnosis: an economic perspective. Support Care Cancer. 2013;21(12):3461-3469. doi:10.1007/s00520-013-1931-3.

19. Girgis A, Lambert S, Johnson C, Waller A, Currow D. Physical, psychosocial, relationship, and economic burden of caring for people with cancer: a review. J Oncol Pract. 2013;9(4):197-202. doi:10.1200/JOP.2012.000690.

20. Pelletier W, Bona K. Assessment of financial burden as a standard of care in pediatric oncology. Pediatr Blood Cancer. 2015;62(S):S619-S631.

21. Delgado-Guay M, Ferrer J, Rieber AG, et al. Financial Distress and Its Associations with Physical and Emotional Symptoms and Quality of Life Among Advanced Cancer Patients. Oncologist. 2015;20(9):1092-1098. doi:10.1634/theoncologist.2015-0026.

22. Mercadante S, Aielli, Adile C. et al. Financial distress and its impact on symptom expression in advanced cancer patients. Support Care Cancer 29, 485-490 (2021). https://doi.org/10.1007/s00520020-05507-9.

23. Falconier MK, Kuhn R. Dyadic Coping in Couples: A Conceptual Integration and a Review of the Empirical Literature. Front Psychol. 2019;10:571. Published 2019 Mar 26. doi:10.3389/fpsyg.2019.00571.

24. Bruera E, Kuehn N, Miller MJ et al. The Edmonton Symptom Assessment System' (ESAS): a simple method for the assessment of palliative care patients. J Palliat Care 1991;7:6 PubMed: 1714502.

25. Zabora J, BrintzenhofeSzoc K, Jacobsen P, et al. A new psychosocial screening instrument for use with cancer patients. Psychosomatics 2001;42:241-6.

26. Radloff LS. The CES-D scale: A new self-report depression scale for research in the general population. Applied Psychological Measurement 1977; 1:385-401.

27. Hays RD, Bjorner JB, Revicki DA, Spritzer KL, Cella Development of physical and mental health summary scores from the patient-reported outcomes measurement information system (PROMIS) global items. Qual Life Res 2009;18:7:873-880 PubMed: 19543809. 
28. Arden-Close E, Moss-Morris R, Dennison L, Bayne L, Gidron Y. The Couples' Illness Communication Scale (CICS): development and validation of a measure assessing illness-related couple communication. Br J Health Psychol 2010;15:543-559.

29. Kenny DA, Kashy DA, Cook WL: Dyadic data analysis. New York, Guilford Press, 2006.

30. Barbaret C, Delgado-Guay MO, Sanchez S, et al. Inequalities in Financial Distress, Symptoms, and Quality of Life Among Patients with Advanced Cancer in France and the U.S. Oncologist. 2019;24(8):1121-1127. doi:10.1634/theoncologist.2018-0353.

31. Lopez G, Milbury K, Chen M, Li Y, Bruera E, Cohen L. Couples' symptom burden in oncology care: perception of self and the other. Support Care Cancer. 2019;27(1):139-145. doi:10.1007/s00520-0184298-7.

32. Langer SL, Brown JD, Syrjala KL. Intrapersonal and interpersonal consequences of protective buffering among cancer patients and caregivers. Cancer. 2009;115(18 Suppl):4311-4325. doi:10.1002/cncr.24586.

33. Manne SL, Norton TR, Ostroff JS, Winkel G, Fox K, Grana G. Protective buffering and psychological distress among couples coping with breast cancer: The moderating role of relationship satisfaction. $J$ Fam Psychol. 2007;21(3):380-388. doi:10.1037/0893-3200.21.3.380.

34. Fenn KM, Evans SB, McCorkle R, et al. Impact of financial burden of cancer on survivors' quality of life. J Oncol Pract. 2014;10(5):332-338

doi:10.1200/JOP.2013.001322.

35. Tarnasky AM, Tran GN, Nicolla J, Friedman FAP, Wolf S, Troy JD et al. Mobile Application to Identify Cancer Treatment-Related Financial Assistance: Results of a Randomized Controlled Trial. Journal of Clinical Oncology Practice 2021; PMID: 33797952; DOI 10.1200/OP.20.00757.

36. Nipp RD, Lee H, Gorton E, et al. Addressing the Financial Burden of Cancer Clinical Trial Participation: Longitudinal Effects of an Equity Intervention. Oncologist. 2019;24(8):1048-1055. doi:10.1634/theoncologist.2019-0146.

\section{Tables}

Table 1. Participant demographics and characteristics 
Patients Partners

Mean (SD) Min-Max Mean (SD) Min-Max

$\chi_{t}^{2}$ or Paired

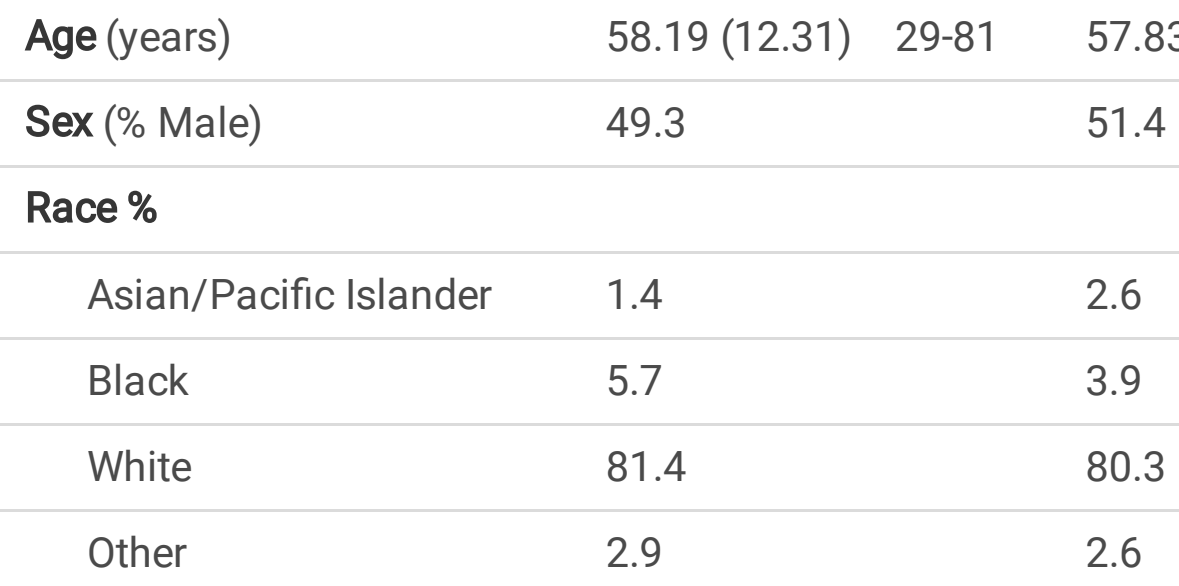

\section{Ethnicity \%}

Hispanic

8.6

10.5

\section{Employment \%}

Full-time employed

33.8

50.7

Part-time employed

11.3

4.0

Retired

46.5

36.0

Unemployed

8.4

9.3

Education \%

Graduate degree

15.3

21.3

Bachelor's Degree

29.1

28.3

Some college

37.5

29.6

High school graduate

16.7

14.7

Some high school

1.4

4.0

Diagnosis \%

\begin{tabular}{llllll} 
Brain Tumor & 43.4 & & - \\
\hline Lung Cancer & 56.6 & - & \\
\hline Time since diagnosis (yrs) & $2.76(4.6)$ & $0-27$ & - & \\
\hline Emotional Distress & & & & & \\
\hline $\begin{array}{c}\text { BSI-18 Anxiety } \\
\text { Symptoms }\end{array}$ & $0.31(0.34)$ & $0-1.67$ & $0.458(0.45)$ & $0-1.67$ & $-2.77^{*}$ \\
\hline
\end{tabular}




\begin{tabular}{|c|c|c|c|c|}
\hline CES-D Depression & 10.69 (7.3) 0 - 37 & $11.75(9.9)$ & $0-54$ & -1.10 \\
\hline CES-D Cases $\geq 16(\%)$ & $\mathrm{n}=15(20.5 \%)$ & $\mathrm{n}=16(21.1 \%)$ & & 3.61 \\
\hline $\begin{array}{l}\text { PROMIS-10 Physical } \\
\text { QOL }\end{array}$ & $\begin{array}{l}43.74(9.3) \quad 19.9- \\
61.9\end{array}$ & $\begin{array}{l}48.47(10.7) \\
67.7\end{array}$ & $4.8-$ & $-3.09 *$ \\
\hline
\end{tabular}

\begin{tabular}{|c|c|c|c|}
\hline OMIS-10 Mental QOL & $50.51(8.8)$ & $31.3-67.6$ & $\begin{array}{l}51.25(9.3) \\
67.6\end{array}$ \\
\hline
\end{tabular}

\section{Relationship}

CICS

$16.74(3.1)$

$15.67(3.6)$

$2.38^{*}$

${ }^{*} \mathrm{p}<.05,{ }^{* \star \mathrm{p}}<.001$.

Note, Quality of Life (QOL), CES-D $\geq 16$ indicates clinical levels of depression, PROMIS-10 measures, higher scores indicate more of the concept being measured. CICS, Couples Illness Communication Scale, higher scores indicate greater ratings of ease of illness communication within the couple. Possible scores range from 4 to 20 .

Table 2. Patient and spousal caregiver self-report of symptoms on ESAS

\begin{tabular}{llcccll} 
& Patients & \multicolumn{6}{l}{ Spousal Caregivers } \\
& Mean (SD) & Rank & Mean (SD) & Rank & Correlation & Paired t \\
\hline Pain & $2.49(2.64)$ & 5 & $1.49(2.14)$ & 8 & 0.16 & $2.58^{\star}$ \\
\hline Fatigue & $4.00(2.71)$ & 1 & $2.79(2.56)$ & 3 & 0.70 & $2.79^{\star}$ \\
\hline Nausea & $1.16(2.07)$ & 10 & $0.34(1.33)$ & 12 & 0.29 & $3.30^{\star}$ \\
\hline Depression & $1.05(1.88)$ & 11 & $1.43(2.20)$ & 9 & 0.09 & -1.09 \\
\hline Anxiety & $1.64(2.23)$ & 9 & $2.41(2.66)$ & 5 & 0.09 & $-2.04^{\star}$ \\
\hline Drowsiness & $2.20(2.59)$ & 8 & $1.65(2.31)$ & 7 & 0.06 & 1.23 \\
\hline Appetite & $3.00(2.90)$ & 4 & $2.13(2.71)$ & 6 & 0.15 & $2.00^{\star}$ \\
\hline Well Being & $3.07(2.56)$ & 3 & $2.76(2.72)$ & 4 & 0.19 & 0.66 \\
\hline Shortness of Breath & $2.45(2.84)$ & 6 & $0.66(1.37)$ & 11 & $0.26^{\star}$ & $5.30^{\star *}$ \\
\hline Sleep & $3.96(2.50)$ & 2 & $3.79(2.69)$ & 1 & 0.05 & 0.17 \\
\hline Financial Distress & $2.32(2.81)$ & 7 & $2.81(3.11)$ & 2 & $0.53^{\star *}$ & -1.57 \\
\hline Spiritual Pain & $0.69(1.42)$ & 12 & $1.19(2.41)$ & 10 & $0.30^{\star}$ & -0.09
\end{tabular}

${ }^{*} \mathrm{p}<.05,{ }^{* \star} \mathrm{p}<.001$. Edmonton Symptom Assessment Scale (ESAS) 
Table 3. Associations among self-report financial distress, psychological symptoms, illness communication, and quality of life

\begin{tabular}{|c|c|c|c|c|c|c|}
\hline & $\begin{array}{l}\text { Financial } \\
\text { Distress }\end{array}$ & $\begin{array}{l}\text { BSI-18 } \\
\text { Anxiety }\end{array}$ & $\begin{array}{l}\text { CESD - } \\
\text { Depression }\end{array}$ & $\begin{array}{l}\text { Physical } \\
\text { QOL }\end{array}$ & $\begin{array}{l}\text { Mental } \\
\text { QOL }\end{array}$ & CICS \\
\hline Financial Distress & $.533^{\star \star *}$ & $.311 *$ & .391 ** & $-.377 * \star$ & $-.442^{\star \star}$ & -.103 \\
\hline BSI-18 Anxiety & $.290 *$ & .227 & $.649 \star \star$ & $-.374 * \star$ & $-.514^{\star \star}$ & $-.371^{\star \star}$ \\
\hline CESD-Depression & $.292^{\star}$ & $.599 * \star$ & $.418^{\star \star}$ & $-.593 \star \star$ & $-.607^{\star \star}$ & $-.382^{\star \star}$ \\
\hline Physical QOL & $-.254^{\star}$ & $-.334 * \star$ & $-.477^{\star \star}$ & .106 & $.728 * \star$ & $.271^{\star}$ \\
\hline Mental QOL & -.159 & $-.489 \star \star$ & $-.534 \star \star$ & $.716 \star \star$ & $.377^{\star}$ & $.326 *$ \\
\hline $\begin{array}{l}\text { CICS- Illness } \\
\text { Communication }\end{array}$ & -.170 & $-.239 *$ & -.210 & .041 & .113 & $.361^{\star}$ \\
\hline
\end{tabular}

${ }^{*} \mathrm{p}<.05,{ }^{*} \mathrm{p}<.001$. Note, Patient below the diagonal, Spousal Caregiver, above the diagonal, Partial correlation of patient and partner self-report on the diagonal. BSI-18 Anxiety, anxiety subscale of brief symptom inventory-18. CESD, Center for Epidemiological Studies Depression Scale. QOL, Quality of Life. CICS, Couples IIIness Communication Scale.

Table 4. Patient self-report rating and spousal caregiver proxy-report of symptoms on ESAS 
Patient Self-Report Caregiver Proxy-Report

of Patient Symptoms
Caregiver

Response Style

\begin{tabular}{|c|c|c|c|c|c|c|}
\hline & $\begin{array}{l}\text { Mean (SD) } \\
\text { Rank }\end{array}$ & $\begin{array}{l}\text { Mean (SD) } \\
\text { Rank }\end{array}$ & & Correlation & $\begin{array}{l}\text { Paired } \\
\text { T-test }\end{array}$ & \\
\hline Pain & $2.49(2.64)$ & $3.49(3.00)$ & 3 & $0.66^{* *}$ & $-3.31^{* *}$ & Overreport $^{+}$ \\
\hline Fatigue & $\begin{array}{l}4.00(2.71) \\
1\end{array}$ & $5.01(2.78)$ & 1 & $0.50^{\star \star}$ & $-2.84^{*}$ & Overreport $^{+}$ \\
\hline Nausea & $\begin{array}{l}1.16(2.07) \\
10\end{array}$ & $2.04(2.57)$ & 11 & $0.71^{* *}$ & $-3.97^{\star *}$ & Overreport \\
\hline Depression & $\begin{array}{l}1.05(1.88) \\
11\end{array}$ & $2.17(2.35)$ & 10 & $0.38^{\star \star}$ & $-3.82^{\star *}$ & Overreport $^{+}$ \\
\hline Anxiety & $\begin{array}{l}1.64(2.23) \\
9\end{array}$ & $2.73(2.50)$ & 7 & $0.52^{* \star}$ & $-4.39^{\star *}$ & Overreport $^{+}$ \\
\hline Drowsiness & $\begin{array}{l}2.20(2.59) \\
8\end{array}$ & $2.87(2.81)$ & 5 & $0.42^{* \star}$ & $-2.01^{*}$ & Overreport \\
\hline Appetite & $\begin{array}{l}3.00(2.90) \\
4\end{array}$ & $2.61(2.47)$ & 6 & $0.27^{\star \star}$ & 0.81 & Accurate \\
\hline Well Being & $\begin{array}{l}3.07(2.56) \\
3\end{array}$ & $3.29(2.43)$ & 4 & 0.12 & -0.67 & Accurate \\
\hline $\begin{array}{l}\text { Shortness of } \\
\text { Breath }\end{array}$ & $\begin{array}{l}2.45(2.84) \\
6\end{array}$ & $2.39(2.84)$ & 8 & $0.63^{* *}$ & 0.25 & Accurate \\
\hline Sleep & $\begin{array}{l}3.96(2.50) \\
2\end{array}$ & $4.18(2.52)$ & 2 & $0.40^{\star *}$ & -0.95 & Overreport \\
\hline $\begin{array}{l}\text { Financial } \\
\text { Distress }\end{array}$ & $\begin{array}{l}2.32(2.81) \\
7\end{array}$ & $2.21(2.70)$ & 9 & 0.23 & 0.54 & Accurate \\
\hline Spiritual Pain & $\begin{array}{l}0.69(1.42) \\
12\end{array}$ & $1.14(2.00)$ & 12 & $0.36^{* \star}$ & $2.38^{*}$ & Overreport \\
\hline
\end{tabular}

${ }^{*} \mathrm{p}<.05,{ }^{*} \mathrm{p}<.001$, Edmonton Symptom Assessment Scale (ESAS), ${ }^{+}$clinically significant difference as indicated by a full point difference between caregiver and patient

Table 5. Spouse self-report and patient proxy report of partner symptoms on ESAS 


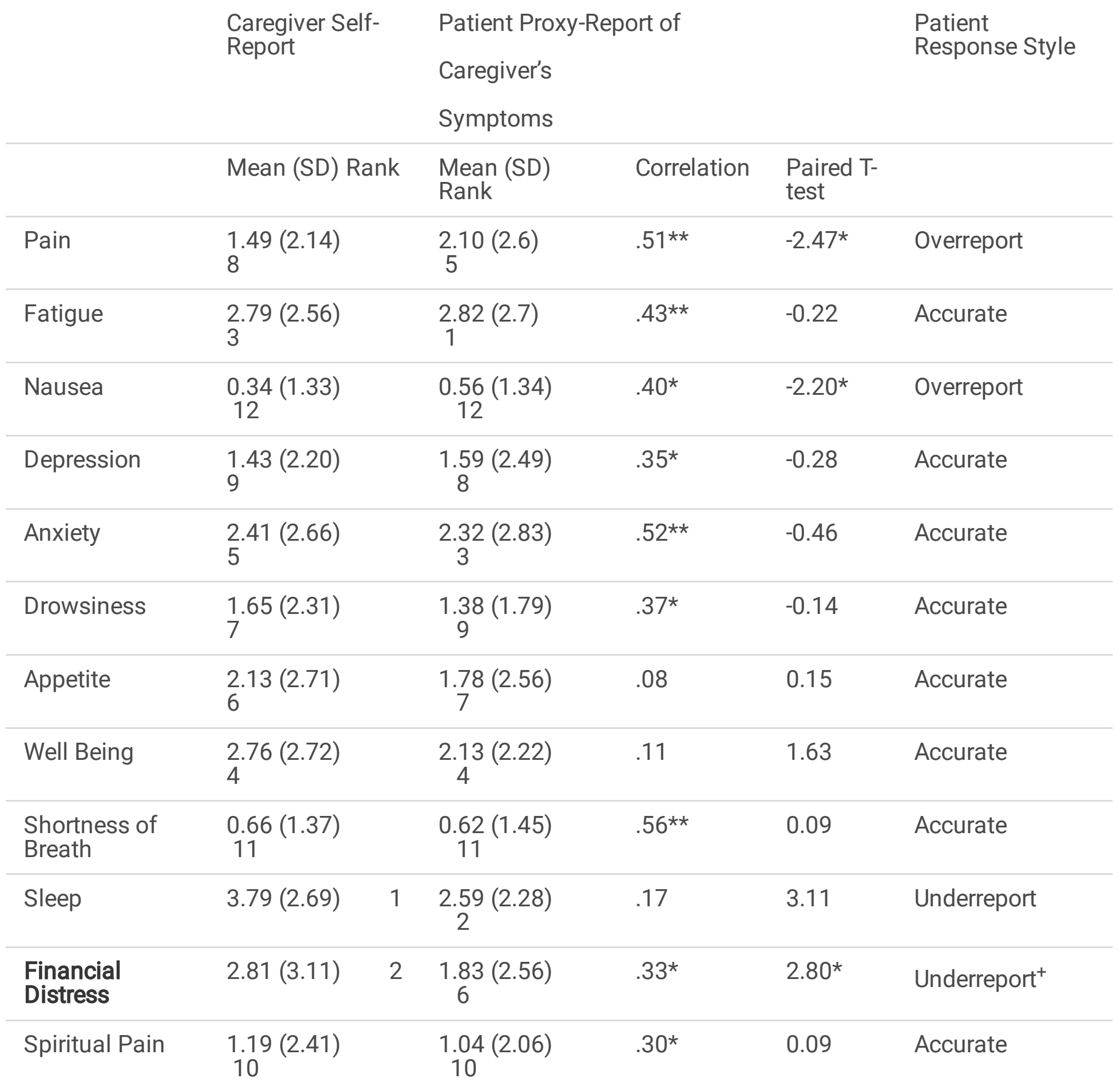

${ }^{*} \mathrm{p}<.05,{ }^{*} \mathrm{p}<.001$, Mean values reported for entire sample, correlation and t-test statistics based on data available for both patient and partner, ${ }^{+}$clinically significant difference as indicated by a full point difference between caregiver and patient

\section{Figures}

\section{Figure 1}


Relative Symptom Severity among Patients and Spouses. Note, ${ }^{*} \mathrm{p}<.05$, Paired sample t-test demonstrate no significant difference between patients and their spouses on Financial Distress (FD)

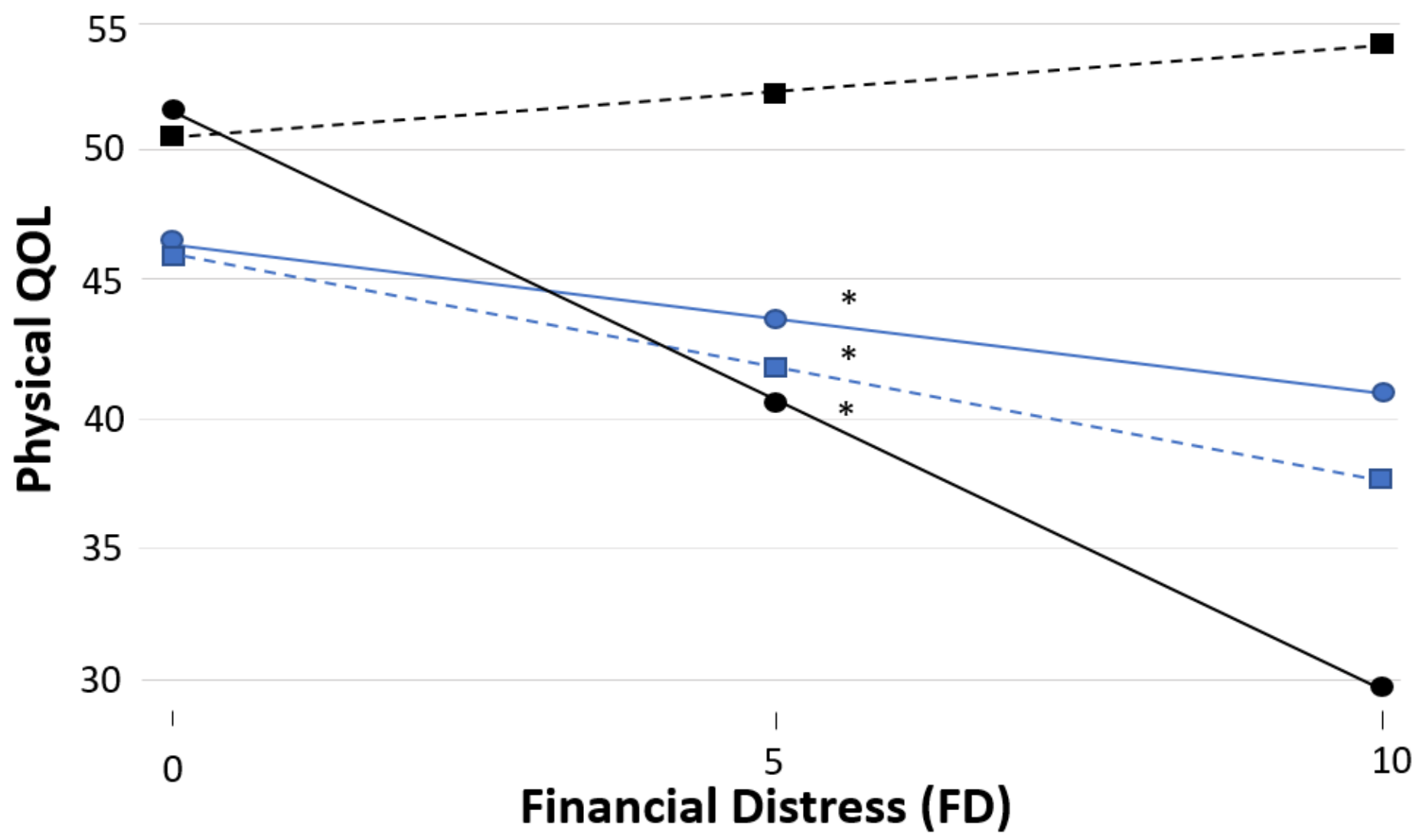

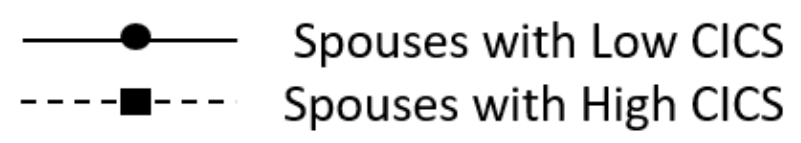

Figure 2

Association between FD and Physical QOL of patients and spousal caregivers at low and high levels of couples illness communication scale (CICS). Note: * indicates significant slope at $p<.05$; Quality of Life (QOL); Couples Illness Communication Scale (CICS); High = 1 SD above the mean; Low $=1$ SD below the mean. While patients, regardless of illness communication levels, and spouses with low illness communication levels had a negative association between FD with physical QOL, spouses with high illness communication did not demonstrate a negative association.

\section{Supplementary Files}

This is a list of supplementary files associated with this preprint. Click to download.

- BrueraCOIForm520.pdf

- ChenCOIForm520.pdf

- ChoCOIForm520.pdf 
- JLKrollCOIForm520.pdf

- KimCOIForm520.pdf

- KrollFDCouplesCoverLetter.pdf

- MilburyCOIForm520.pdf

- SmithCOIForm520.pdf

- WeathersCOIForm520.pdf 